\title{
STONE-WEIERSTRASS THEOREM
}

\author{
GUY LAVILLE and IVAN P. RAMADANOFF \\ Université de Caen \\ Département de Mathématiques \\ Esplanade de la Paix \\ 14032 Caen Cedex, France
}

\begin{abstract}
It will be shown that the Stone-Weierstrass theorem for Clifford-valued functions is true for the case of even dimension. It remains valid for the odd dimension if we add a stability condition by principal automorphism.
\end{abstract}

Introduction. Recall the classical Stone-Weierstrass theorem: let $Y$ be a metric space, $\mathcal{C}(Y ; \mathbb{R})$ the set of all continuous functions from $Y$ in $\mathbb{R}, B \subset \mathcal{C}(Y ; \mathbb{R})$ a subset such that $B$ contains the constant function 1 and separates the points of $Y$. Then the algebra $A_{B}(Y ; \mathbb{R})$, generated by $B$ is dense in $\mathcal{C}(Y ; \mathbb{R})$ for the topology of the uniform convergence on every compact.

It is well-known that if one substitutes the field $\mathbb{R}$ by $\mathbb{C}$, then an additional hypothesis is needed, namely: $B$ should be stable with respect to complex conjugation. In case we are omitting this hypothesis and if we take, for example, $Y$ to be an open subset of $\mathbb{C}$ and $Y=\{1, z\}$, then we will get the algebra of holomorphic functions.

Let us mention that the case of functions taking values in the quaternion field is known [2] and it is analogous to the real case.

Here, we will investigate the situation when $\mathbb{R}$ is replaced by $\mathbb{R}_{p, q}$ - a universal Clifford algebra of $\mathbb{R}^{n}, n=p+q$, with a quadratic form of signature $(p, q)$. This study is motivated by the theory of monogenic functions [1]. The present paper is organized as follows: in Section 1 we will recall some notations usually employed in Clifford algebras. Section 2 will deal with some elements of combinatorics. The essential part of the paper is Section 3 in which we give a formula allowing to compute the scalar part of a given Clifford number. As an application of this formula, we are able to prove in Section 4 the following Stone-Weierstrass theorem for $\mathcal{C}\left(Y ; \mathbb{R}_{p, q}\right)$ :

THEOREM. Let $Y$ be a metric space and $\mathcal{C}\left(Y ; \mathbb{R}_{p, q}\right)$ the set of all continuous functions from $Y$ to $\mathbb{R}_{p, q}$. Let $B \subset \mathcal{C}\left(Y, \mathbb{R}_{p, q}\right)$ be such that $B$ contains the constant function 1

1991 Mathematics Subject Classification: Primary 30G35; Secondary 32K99.

The paper is in final form and no version of it will be published elsewhere. 
and separates the points of $Y$. If $p+q$ is odd, suppose in addition that $B$ is stable with respect to the principal automorphism $*$. Then, the algebra $A_{B}\left(Y ; \mathbb{R}_{p, q}\right)$, generated by $B$, is dense in $\mathcal{C}\left(Y ; \mathbb{R}_{p, q}\right)$ for the topology of uniform convergence on compact sets.

1. Notations. In a Clifford algebra $\mathbb{R}_{p, q}=C_{0} \oplus C_{1} \oplus \ldots \oplus C_{n}$, with $n=p+q$, the spaces $C_{0}, C_{1}, \ldots, C_{n}$ are supposed to be of respective basis $\{1\},\left\{e_{1}, e_{2}, \ldots, e_{n}\right\}$, $\left\{e_{i j}\right\}_{i<j}, \ldots,\left\{e_{i_{1} \ldots i_{k}}\right\}_{i_{1}<i_{2}<\cdots<i_{k}}, \ldots,\left\{e_{1.2 \ldots n}\right\}$, where $\left(i_{1}, \ldots, i_{k}\right)$ is a multiindex with $i_{1}, \ldots, i_{k} \in\{1, \ldots, n\}, 1 \leq i_{1}<\ldots<i_{k} \leq n$. The algebra obeys to the laws:

$$
\begin{cases}e_{i}^{2}=1, & i=1, \ldots, p, \\ e_{i}^{2}=-1, & i=p+1, \ldots, n, \\ e_{i} e_{j}=-e_{j} e_{i}, & i \neq j, \\ e_{i_{1} \ldots i_{k}}=e_{i_{1}} e_{i_{2}} \cdots e_{i_{k}}, & \text { for } i_{1}<i_{2}<\ldots<i_{k} .\end{cases}
$$

We will make use of the decomposition of a Clifford number a in its scalar (real) part $\langle a\rangle_{0}$, its 1-vector $\langle a\rangle_{1} \in C_{1}$, its bivector part $\langle a\rangle_{2} \in C_{2}$, etc ... up to its pseudo-scalar part $\langle a\rangle_{n} \in C_{n}$, i.e:

$$
a=\langle a\rangle_{0}+\langle a\rangle_{1}+\cdots+\langle a\rangle_{n}
$$

where,

$$
\langle a\rangle_{k}=\sum_{\substack{J \\|J|=k}} a_{J} e_{J}
$$

$J=\left(j_{1}, \ldots, j_{k}\right)$ is a multiindice and $|J|=k, \quad e_{J}=e_{j_{1}} \cdots e_{j_{k}}$.

Recall that the principal involution ${ }_{*}$, the anti-involution ${ }^{*}$ and the reversion $\sim$ act on $a \in \mathbb{R}_{0, n}$ as follows:

$$
\begin{aligned}
& a_{*}=\sum_{k=0}^{n}(-1)^{k}\langle a\rangle_{k} \\
& a^{*}=\sum_{k=0}^{n}(-1)^{\frac{k(k+1)}{2}}\langle a\rangle_{k} \\
& a^{\sim}=\sum_{k=0}^{n}(-1)^{\frac{k(k-1)}{2}}\langle a\rangle_{k}
\end{aligned}
$$

Now, define

$$
e^{i}=\left\{\begin{array}{lll}
e_{i}, & \text { if } \quad 1 \leq i \leq p \\
-e_{i}, & \text { if } \quad p+1 \leq i \leq p+q
\end{array}\right.
$$

and $e^{J}=e^{j_{k}} \cdots e^{j_{1}}$.

2. Some combinatorics. Let us study the partition of the set $\{1, \ldots, n\}$ in two strictly ordered subsets: $I=\left\{i_{1}, \ldots, i_{k}\right\}$ and $J=\left\{j_{1}, \ldots, j_{p}\right\}$. As for as the relative position of $J$ with respect to $I$ is concerned, we have different possible cases: $J \cap I=\phi$; just one $j_{\alpha}$ belongs to $I ; \ldots ; \ell$ among the $j_{\alpha}^{\prime} s$ belong to $I ; \ldots$; the largest possible number of $j_{\alpha}^{\prime} s$ belongs to $I$. It is easy to compute the cardinals of the corresponding sets: 
For the first case, the cardinal is $C_{n-k}^{p} C_{k}^{\sup \{0, p-(n-k)\}}$. If just one $j_{\alpha}$ belongs to $I$, then we will have $C_{n-k}^{p-1} C_{k}^{\sup \{0, p-(n-k)\}+1}$ and so on ... In the last case, we will get $C_{n-k}^{0} C_{k}^{\inf \{p, k\}}$.

Now, recall the following result which is well-known in classical probability theory [3]:

Lemma 1. For every $k, 0 \leq k \leq n$ :

$$
\sum_{\ell=\sup \{0, p-(n-k)\}}^{\inf \{p, k\}} C_{n-k}^{p-\ell} C_{k}^{\ell}=C_{n}^{p} .
$$

In fact, this lemma will not be used here, but its elementary proof, which will be given below, is a source of inspiration for the next result (Lemma 2).

Pr o of. For every $k, 0 \leq k \leq n$, one has $(1+x)^{n-k}(1+x)^{k}=(1+x)^{n}$, which involves

$$
\sum_{\ell=0}^{k}(1+x)^{n-k} C_{k}^{\ell} x^{\ell}=\sum_{p=0}^{n} C_{n}^{p} x^{p},
$$

and again:

$$
\sum_{\ell=0}^{k} \sum_{n=0}^{n-k} C_{n-k}^{n} x^{n} \quad C_{k}^{\ell} x^{\ell}=\sum_{p=0}^{n} C_{n}^{p} x^{p} .
$$

Let us set $n+\ell=p$, i.e. $n=p-\ell$. Then the double sum is equal to

$$
\sum_{\ell=0}^{k} \sum_{p=\ell}^{n-k+\ell} C_{n-k}^{p-\ell} C_{k}^{\ell} x^{p}=\sum_{p=0}^{n} \sum_{\ell=\sup \{0, p-(n-k)\}}^{\inf \{p, k\}} C_{n-k}^{p-\ell} C_{k}^{\ell} x^{p} .
$$

It just remains to identify the coefficients of $x^{p}$. Now, we are in a position to formulate and prove the following:

Lemma 2.

$$
\sum_{p=0}^{n} \sum_{\ell=\sup \{0, p-(n-k)\}}^{\inf \{p, k\}}(-1)^{p k+\ell} C_{n-k}^{p-\ell} C_{k}^{\ell}= \begin{cases}0, & \text { if } 1 \leq k \leq n-1 \\ 0, & \text { if } k=n, n \text { even } \\ 2^{n}, & \text { if } k=n, n \text { odd } \\ 2^{n}, & \text { if } k=0 .\end{cases}
$$

Proof. Start from

$$
\begin{aligned}
\left(1+(-1)^{k} x\right)^{n-k}(1+ & \left.(-1)^{k+1} x\right)^{k}= \\
& =\sum_{\ell=0}^{k}\left(1+(-1)^{k} x\right)^{n-k}(-1)^{(k+1) \ell} C_{k}^{\ell} x^{\ell}= \\
& =\sum_{\ell=0}^{k} \sum_{n=0}^{n-k}(-1)^{k n} C_{n-k}^{n} x^{n}(-1)^{(k+1) \ell} C_{k}^{\ell} x^{\ell}= \\
& =\sum_{p=0}^{n} \sum_{\ell=\sup \{0, p-(n-k)\}}^{\inf \{p, k\}}(-1)^{p k+\ell} C_{n-k}^{p-\ell} C_{k}^{\ell} x^{p},
\end{aligned}
$$


because $k n+(k+1) \ell=p k+\ell$. Thus it is enough to set $x=1$ and remark that:

$$
\left(1+(-1)^{k}\right)^{n-k}\left(1+(-1)^{k+1}\right)^{k}= \begin{cases}2^{n}, & \text { if } k=0 \\ 0, & \text { if } 1 \leq k \leq n-1 \\ 2^{n}, & \text { if } k=n, n \text { odd } \\ 0, & \text { if } k=n, n \text { even }\end{cases}
$$

\section{A formula for the real part of $a \in \mathbb{R}_{p, q}$.}

Lemma 3. For every multiindex $J$, we have $e_{J} e^{J}=1$.

Lemma 4. Let $I=\left(i_{1}, \ldots, i_{k}\right), \quad|I|=k . \quad J=\left(j_{1}, \ldots, j_{p}\right), \quad|J|=p$ there is the following equality

$$
\sum_{p=0}^{n} \sum_{|J|=p} e_{J} e_{I} e^{J}= \begin{cases}2^{n} & \text { if } k=0 \text { or } \text { if } k=n \text { with } n \text { odd } \\ 0 & \text { in other cases }\end{cases}
$$

Proof. Decompose the sum

$$
\sum_{|J|=p} e_{j} e_{I} e^{J}
$$

following the relative position of $J$ with respect to $I$. If $J \cap I=\phi$ we have $C_{n-k}^{p} C_{k}^{0}$ such possibilities and the anticommutation gives $(-1)^{p k}$.

If only one $j_{\alpha} \in I$ we have $C_{n-k}^{p-1} C_{k}^{1}$ such possibilites and the anticommutation gives $(-1)^{(p-1) k}(-1)^{k-1}$ and so on, $\ldots$, if $\ell j_{\alpha} \in I$ we have $C_{n-k}^{(p-\ell) k} C_{k}^{\ell}$ such possibilities and the commutation gives $(-1)^{(p-\ell) k}(-1)^{\ell(k-1)}$.

The sum is equal to

$$
\sum_{\ell=\sup \{0, p-(n-k)\}}^{\inf \{p, k\}}(-1)^{(p-\ell) k}(-1)^{\ell(k-1)} C_{n-k}^{p-\ell} C_{k}^{\ell} e_{I}
$$

Thus we could apply lemma 2 and the result follows.

The next result is a formula for the scalar part of a Clifford number.

TheOrem 1. Let $a \in \mathbb{R}_{p, q}$. Then:

a) if $n$ is even,

$$
\langle a\rangle_{0}=\frac{1}{2^{n}} \sum_{p=0}^{n} \sum_{|J|=p} e_{J} a e^{J} .
$$

b) if $n$ is odd,

$$
\langle a\rangle_{0}=\frac{1}{2^{n+1}} \sum_{p=0}^{n} \sum_{|J|=p} e_{J} a e^{J}+\frac{1}{2^{n+1}} \sum_{p=0}^{n} \sum_{|J|=p} e_{J} a_{*} e^{J} .
$$

Proof. When $a \in \mathbb{R}_{0, n}$, then

$$
a=\sum_{k=0}^{n} \sum_{|I|=k} a_{I} e_{I}
$$


where $I=\left(i_{1}, \ldots, i_{k}\right), \quad 1 \leq i_{1}<i_{2}<\ldots<i_{k} \leq n$. Take the sum

$$
\sum_{p=0}^{n} \sum_{|J|=p} e_{J} a e^{J}=\sum_{J} \sum_{I} a_{I} e_{J} e_{I} e^{J} .
$$

Now, apply lemma 4:

a) if $n$ is even, one gets:

$$
\sum_{p=0}^{n} \sum_{|J|=p} e_{J} a e^{J}=2^{n}\langle a\rangle_{0}
$$

b) if $n$ is odd, one has:

$$
\sum_{p=0}^{n} \sum_{|J|=p} e_{J} a e^{J}=2^{n}\langle a\rangle_{0}+2^{n}\langle a\rangle_{n} .
$$

But, in the case when $n$ is odd, $\left\langle a_{*}\right\rangle_{n}=(-1)^{n}\langle a\rangle_{n}=-\langle a\rangle_{n}$. Thus, we get the part b) of the theorem.

Rem ark. For $n=1$, the preceding formula becomes to

4 Re $a=(a-i a i)+(\bar{a}-i \bar{a} i)$ in $\mathbb{R}_{0,1}=\mathbb{C}$ with the classical notations of $\mathbb{C}$.

For $n=2$, this means that $4 R e a=a-i a i-j a j-k a k$ in $\mathbb{R}_{0,2}=\mathbb{H}$ with the classical notations of $\mathbb{H},[2]$.

\section{The Stone-Weierstrass theorem for $\mathcal{C}\left(Y ; \mathbb{R}_{p, q}\right)$.}

TheOREm 3. Let $Y$ be a metric space and $\mathcal{C}\left(Y ; \mathbb{R}_{p, q}\right)$ the set of continuous functions from $Y$ into $\mathbb{R}_{p, q}$. Let $B \subset \mathcal{C}\left(Y ; \mathbb{R}_{p, q}\right)$ be such that $B$ contains the constant function 1 and separates the points of $Y$. When $p+q$ is even, nothing more is supposed. If $p+q$ is odd, suppose $B$ to be stable with respect to the principal involution $*$.

Then, the algebra $A_{B}\left(Y ; \mathbb{R}_{p, q}\right)$, generated by $B$, is dense in $\mathcal{C}\left(Y ; \mathbb{R}_{p, q}\right)$ for the topology of uniform convergence on compact sets.

Proof. Set $A_{B}(Y ; \mathbb{R})$ for the subspace of $A_{B}\left(Y ; \mathbb{R}_{p, q}\right)$ consisting of those functions which take real values. This is a real algebra. Let $A_{B}(Y ; \mathbb{R})_{I}$ be the subspace of $A_{B}\left(Y ; \mathbb{R}_{p, q}\right)$ consisting of the $I$-components of functions from $A_{B}\left(Y ; \mathbb{R}_{p, q}\right)$. Thus, we have $f_{I}=\left\langle f e^{I}\right\rangle_{0}$ and $A_{B}(Y ; \mathbb{R})_{I} \subset A_{B}(Y ; \mathbb{R})$ by theorem 2 .

In this way, $A_{B}(Y ; \mathbb{R})$ satisfies to the hypothesis of the classical Stone-Weierstrass theorem for real functions. The algebra $A_{B}(Y ; \mathbb{R})$ is consequently dense in $\mathcal{C}(Y ; \mathbb{R})$. Finally, one can conclude that:

$$
A_{B}\left(Y ; \mathbb{R}_{p, q}\right)=\bigoplus_{I} A_{B}(Y ; \mathbb{R}) e_{I}
$$

is dense in $\mathcal{C}\left(Y ; \mathbb{R}_{p, q}\right)$.

5. A remark. It should be noted that the computation of the scalar part is strongly related to formulas related to the Hestenes multivector derivative: see [4], chapter 2.

After presenting that work at the Banach Center Jan Cnops indicated to one of us a shorter proof of formulas of theorem 1 . 


\section{References}

[1] R. Delanghe, F. Sommen, V. Souček, Clifford Algebra and Spinor-valued functions, Kluwer.

[2] J. Dugundji, Topology, Allyn and Bacon.

[3] W. Feller, An introduction to the theory of Probability and its applications, J. Wiley.

[4] D. Hestenes, G. Sobczyk, Clifford Algebra to Geometric Calculus, Reidel. 